\title{
Design and Implementation of Cooperative Target Control in the Route of Flight Calibration Test
}

\author{
Pan Gaofeng ${ }^{\mathrm{a}}$, Liu Guoqing ${ }^{\mathrm{b}}$, Xue Jun ${ }^{\mathrm{c}}$ and Su Yan ${ }^{\mathrm{c}}$ \\ Marine Department of Satellite Tracing and Metering, Jiangyin China, 214431 \\ apgfzhy@163.com, bLGQ11@163.com , ‘XueJun@163.com, dSYDR@163.com
}

\begin{abstract}
Keywords: Flight calibration test; Cooperative target; Multi-serial port converter; Truncated control protocol
\end{abstract}

Abstract. Calibration flight is a technical means for the performance and accuracy inspection of TT \& $\mathrm{C}$ equipment. It installs some cooperative objects such as transponder on the aircraft in order to simulate a access between the spacecraft and TT \& C equipment, so as to complete some related test projects. The Calibration flight of sea-based is very different from land-based, because of a large number of test equipment, the wide distribution of band, and the complex work modes, all of these make the number of cooperative target is very large and cause lots of difficulty in the design of control. According to the requirement of the transponder's opening or closing and mode switching, this paper designs a plan for controlling the transponder in the calibration flight route, and it is on the basis of not changing the I/O module of the original control system, a multi-serial port converter and a control protocol have been designed to complete the integration of the transponder and the universal modules, meanwhile realizing the effective control of the transponder under the control of the ARM controller. The plan realizes the efficient collection of the new and old systems, and has some characteristics such as powerful function, reliable performance and simple control. It can improve the efficiency of flight calibration test, and the thought of design has certain reference significance for other related system's transformation.

\section{Introduction}

Flight calibration test is a mission mode for verifying the working performance, working state ,technical and tactical indicators of the shipborne TT\&C equipment. It is a technical means to ensure the equipment has combat effectiveness. In terms of the current level of detection technology, flight calibration test is still the most important method to test. Compared with the land-based TT\&C equipment, the sea-based TT\&C equipment has some features such as a large number of test equipment, wide distribution of band, and complex work modes, if the indicators such as ship rocking isolation must be completed on the sea. Correspondingly, the cooperation targets (such as transponder) on the aircraft also need to include various frequency bands, at the same time, in order to satisfy multiple working modes, the transponder of each frequency band should be matched to multiple modes and can be switched manually or when controlled; meanwhile, in order to ensure the system redundancy, two or more transponders are needed for backup in every band ${ }^{[1]}$.

In the past, the cooperative target's working mode was set before the flight and could not be changed in flight. Even if the data has met the requirement under a certain mode, the mode could only be replaced on the next flight, causing a huge waste of resources. It is badly in need of providing a way to switch modes in flight to improve flight utilization ratio and saving flight costs. In this paper, a number of technologies are used to design a control method for cooperative target in the route of flight calibration test. ARM is used as the controller, and all devices are cascaded on the RS485 bus, multi-serial controllers are used to relay the instruction between ARM controllers, cooperative targets and power modules. it is possible to exert effective control on cooperative target's power-off and mode switching so long as following a custom control protocol. This paper providing a practical and simple method to solve this problem. 


\section{System Design}

The designed system needs to complete functions such as switching mode ,electrifying or switching off in flight ${ }^{[2]}$, and the hierarchical design concept has been applied to implement the scheme of the system. The control strategy is shown in Fig 1.

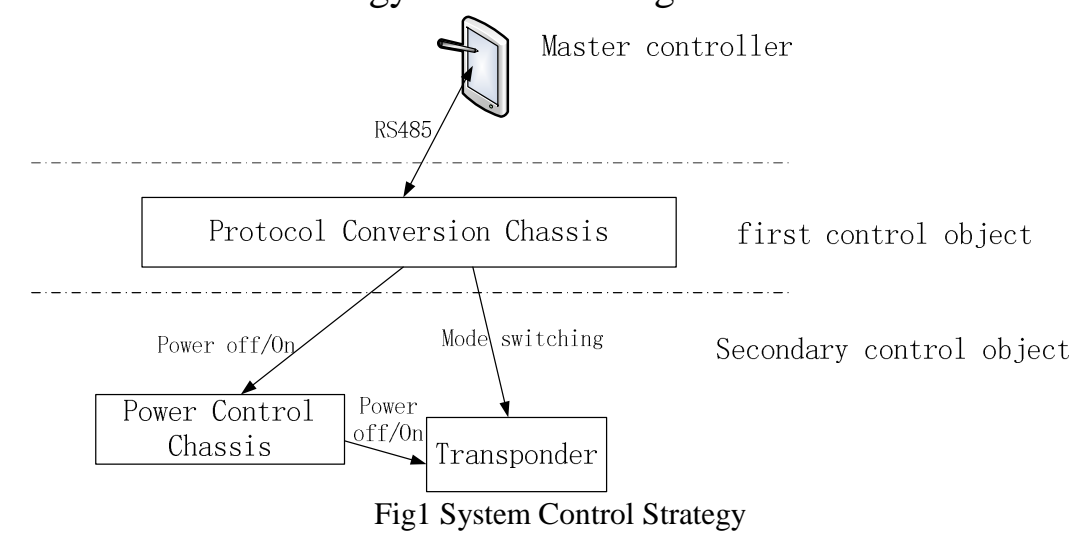

The main controller adopts a portable tablet computer. The instructions are sent to the protocol conversion box through the RS485 bus. The protocol conversion box is a primary control object and is in charge of identifying the power or mode switching instructions, and distributing them to the power control box or the cooperation target. The power control box and the cooperation target are secondary control objects. The power control box controls the relay according to the instructions. A small relay is used to control the relay with large current so as to ensuring the power supply's security and communication security; the cooperation target receiving mode switching instruction to complete mode switching. The system principle is shown in Fig 2. The entire system's design is clear and reasonable, and the interface is standardized, the control is simple, and the cost is low.

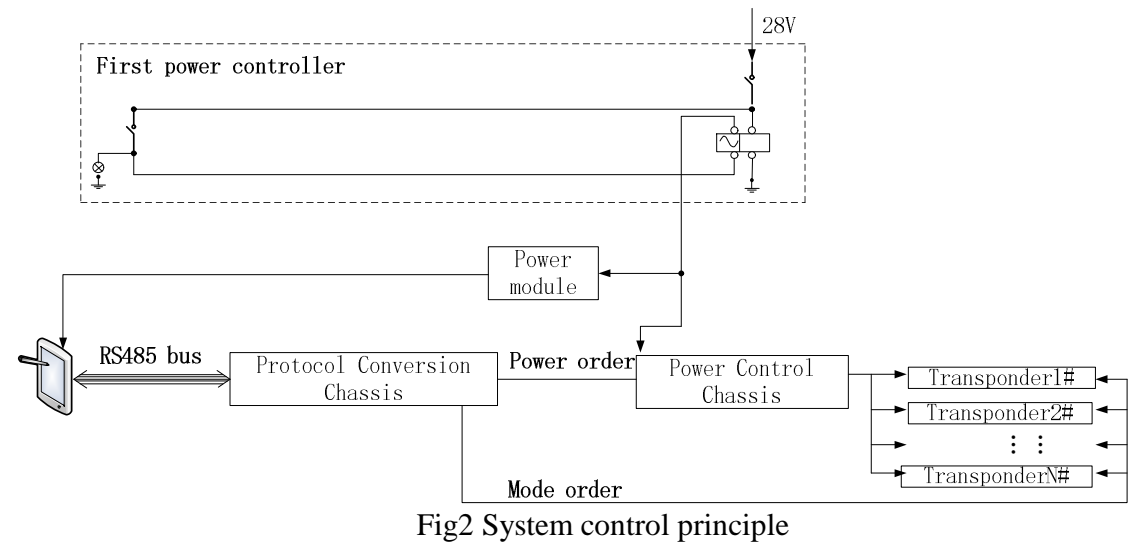

\section{Key Technologies}

Multiple serial controllers. The protocol conversion box is used to complete a conversion of the various subordinate control components, and it is similar to the "middleware" in software. Through this "middleware" of hardware, the old and new devices can be combined together without changing the protocols between the new and old devices. its core is a multi-channel serial board, the principle is shown in Fig 3.

The relay of the original power control box is Advantech's ADAM4017+ module. This module follows the Modbus protocol , and the RS485 interface chip to control the module is selected as SP3485. There are two RS485 serial ports need to be designed, one of them is system bus and needs to receive or feedback ARM controller instructions; the other one is a bus in order to control the power supply box.

In view of the cooperation target's interface is RS422, so the RS422 interface chip selects TI's high-speed driver AM26LV31E and high-speed transmitter AM26LV32E. The isolation chip selects AD's 4-channel receiving isolator ADuM6404 and 4-channel sending isolator ADuM6400, except the 
signal isolation, the driver's and the transmitter's isolation power ISO_VCC and isolation ground ISO_GND can both be isolated too. Not only the signal isolation has been completed, but also the power and the ground are both isolated, so the 4-way RS422 serial ports have been designed.

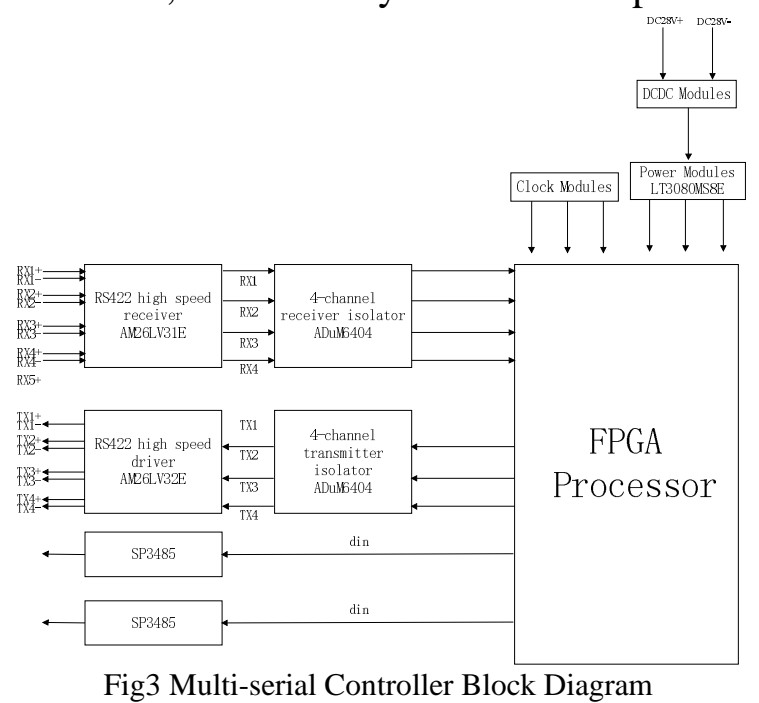

Truncation Control Protocol. Because the cooperation targets are produced by one manufacturer, and the manufacturer adopts a standard control protocol in the industry, it will cause a result that all the control protocols are the same, it will not be able to distinguish the sender of the command if the bussing technique is applied, and all cooperation targets will respond at the same time. In order to overcome this problem, this paper designs a truncation control protocol for data frame processing, as shown in Fig 4.

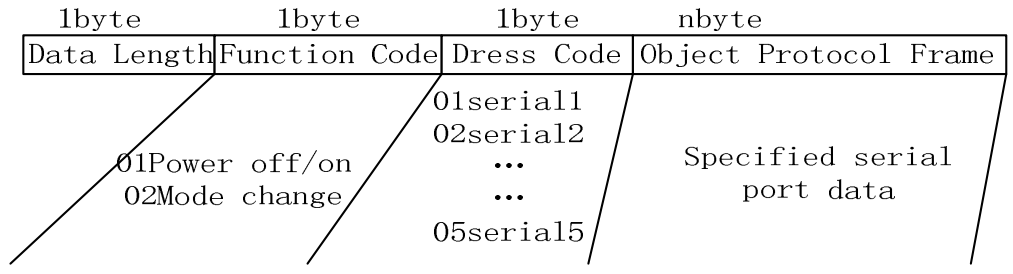

Fig4 Truncated Control Protocol Frame Format

The format of received data is as follows: data length (1byte) + function code (1byte) + address code (1byte) + object protocol frame (n byte). Among them, the data length is an integral multiple of byte number; the address code is used to distinguish the mode switch (01) and power supply control (02); the address code is used to distinguish the corresponding transponder in mode switching; the object protocol frame is not only the protocol of the transponder itself but also the Modbus protocol of the internal power supply box's device. During program processing, the data will be prejudged according to the length of data, which can be used as the basis for the data frame verification and be the number of times which are sent cyclically by the serial ports; the control modes that are distinguished by function codes include the mode control and power control. The instructions sent by the serial ports are distinguished by the address code .For example, if a frame format is 07010307 7B 03 26, it stands for that the data length of the entire frame is 7 bytes, and the control command is mode switching. It needs to execute write commands on the $3 \#$ serial port, the 4 bytes object protocol frame $(07$ 7B 03 26) can be obtained by removing the first 3 bytes data of the entire frame, and it is sent through the $3 \#$ serial port. The format of control feedback frame is as follows: data length (1 byte) + function code ( 1 byte) + address code ( 1 byte $)+$ object feedback frame ( $\mathrm{n}$ byte), Among them, the data length is the length of entire frame data; the function code is the same as the function code of the received data; The address code is the same as the address code of the received data; the object feedback frame is the data frame fed back from the transponder or Modbus device. By truncating the control protocol, controlling devices with different control protocol on the bus is realized, the control circuit is reduced, and the system reliability is improved. 
Software Control Rule Implementation. Due to the existence of changeable control objects, the system composition will also change. At most, two sets of multi-serial controllers and two sets of power control cabinets are required. At most, only one set is required (multi-serial controllers and power control cabinets can be selected. In order to meet the non-professionals' intuitive understanding of controllable objects and avoid the effects of misoperation, it is necessary to facilitate this function without changing the upper computer software.

This paper adopts Ini file to write layout control rules. Its section attributes are number 1-16, which represent each transponder to be controlled. Key attributes include enabling, its value is 0 or 1 ; power-on control instruction, power-off control instruction, the values of the mode switching instruction 01, the mode switching instruction 02 , the mode switching instruction 03 , and the mode switching instruction 04 follow the truncation control protocol frame format, and the values can be empty. The software processing flow is shown in Fig 5.

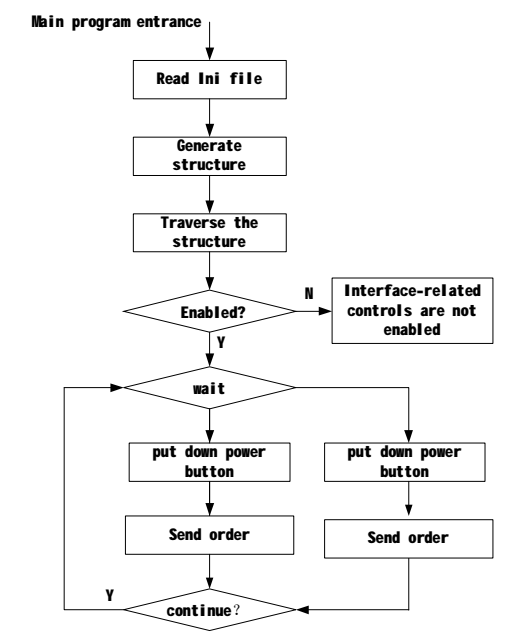

Fig5 Software Processing Ini-File Flow

\section{Conclusions}

This article adopts the hierarchical design concept to complete the system construction, through designing multi-serial controller to complete the efficient integration of the new and old equipment, a reliable transmission of communication instructions has realized according to the truncation control protocol. In the mission of 2017, the aircraft's cooperation objectives were accurately controlled through using this method, the utilization ratio of the route in flight calibration test have improved greatly., it required at least 8 flights to complete one mission in the past, but now 7 flights were enough. It makes important contributions to the flight calibration test. At the same time, the design methods provided in this paper also have a certain significance for the design of other related systems.

\section{Acknowledgements}

This work was financially supported by the Marine Department of Satellite Tracing and Metering.

\section{References}

[1] Pan Gaofeng, Wang Wei. School fly centralized monitoring system based on Modbus protocol [J]. Telemetry and Remote Control, 2008.29-06:59-62.

[2] Pan Gaofeng, Xue Jun et al. Design and implementation of remote wireless control system [J]. Telecommunications Technology, 2012.07:1174-1177. 